Reprod. Nutr. Dévelop., 1987, 27 (1 B), 229-230.

\title{
Etude comparée de la vitesse de vidange du rumen chez le mouton alimenté avec des foins de graminées et de légumineuses
}

Elisabeth GRENET

Laboratoire des Aliments,

I.N.R.A. Theix 63122 Ceyrat, France

Summary. The rate of removal of total and particulate dry matter from the rumen has been measured on fistulated sheep fed one 4-hour meal a day of grass or legume hay. With these hays we observed that the higher the voluntary hay intake, the higher the rumen emptying rate.

Les foins de ray-grass, qu'ils soient précoces ou âgés, se réduisent plus difficilement que les foins de luzerne de même stade en fines particules sous l'action de la mastication lors de l'ingestion par le mouton (Grenet, 1986). Pour ces mêmes foins nous avons aussi mesuré la vitesse de vidange de la matière sèche et des particules du rumen.

Matériel et méthodes. Quatre moutons porteurs d'une canule du rumen ont été alimentés successivement avec 4 foins : une luzerne précoce (LP) ou âgée (LA), et un ray-grass précoce (RGP) ou âgé (RGA) dont la digestibilité de la matière organique et les teneurs en matières azotées et en cellulose brute étaient respectivement : LP, 65,5, 20,1 et 25,8 ; LA, 59,2, 17,2 et 31,9 ; RGP, 75,3, 16,7 et 22,6 ; RGA, 55,3, 9,7 et 33,6. Ces foins ont été offerts en un seul repas par jour, à $8 \mathrm{~h}$, avec enlèvement des refus à $12 \mathrm{~h}$. Le rumen des moutons a été vidé totalement une fois avant la distribution du repas (temps $0 \mathrm{~h}$ ) et $4 \mathrm{~h}$ après (temps $4 \mathrm{~h})$, le contenu a été pesé puis réintroduit dans le rumen après qu'un échantillon représentatif ait été prélevé. La quantité de matière sèche (MS) qui disparaît du rumen pendant les quatre heures qui suivent la distribution du repas a été estimée comme étant égale à : quantité de MS présente à $0 \mathrm{~h}+\mathrm{MS}$ ingérée de 0 à $4 \mathrm{~h}$ - quantité de MS présente dans le rumen à $4 \mathrm{~h}$. La teneur en particules des échantillons est égale à la teneur en MS diminuée de la teneur en substances solubles dans l'eau.

Résultats et discussion. Les quantités ingérées ont été différentes selon les foins (tabl. 1), LP ayant été paradoxalement moins bien consommée que LA, contrairement aux résultats observés sur des animaux alimentés avec deux repas par jour ; cette faible ingestion est certainement due à une appétibilité plus faible de LP que de LA, liée à une plus mauvaise conservation, car la vitesse d'ingestion de LP était plus faible que celle de LA alors que la vitesse de digestion en sachets dans le rumen de LP était supérieure à celle de LA (résultats non publiés). Les quantités de matière fraîche (MF) et de MS présentes dans le rumen à $0 \mathrm{~h}$ sont variables selon les foins ; la quantité de MS est plus élevée pour les foins âgés, notamment LA, que pour les foins précoces. La vitesse de disparition de la MS entre 0 et $4 \mathrm{~h}$ est particulièrement élevée pour LA, un peu moins pour RGP et nettement plus faible pour les deux autres foins. Elle est moins élevée pendant les $20 \mathrm{~h}$ suivantes et diffère peu entre les foins ; elle devient respectivement pour LP, LA et RGP : 2 fois, 4 fois et 3 fois moins rapide, mais elle varie peu pour RGA. La vitesse de disparition des particules du rumen est également différente pour les 
4 foins pendant les 4 heures qui suivent la distribution du repas. Pour LA elle est très élevée $(129 \mathrm{~g} / \mathrm{h})$, supérieure aux autres foins, et elle est la plus faible pour RGA $(17 \mathrm{~g} / \mathrm{h})$. Pendant le reste de la journée les vitesses de disparition des particules de 4 foins varient entre 28 et $40 \mathrm{~g} / \mathrm{h}$ et sont d'autant plus faibles qu'elles étaient plus élevées entre 0 et $4 \mathrm{~h}$.

TABL. 1. - Changements intervenant dans le rumen après le repas. MF : matière fraîche ; MS : matière sèche; NDF : (neutral detergent fibre) parois totales Van Soest ; $P$ : particules.

\begin{tabular}{|c|c|c|c|c|c|}
\hline $\begin{array}{l}\text { Quantité } \\
\text { ingérée }(\mathrm{g} / \mathrm{j}) \text { : }\end{array}$ & $\begin{array}{l}\text { MF } \\
\text { MS } \\
\text { NDF } \\
P\end{array}$ & $\begin{array}{cc} & \text { LP } \\
1450 \pm 78 \\
1270 \pm 70 \\
502 \pm 27 \\
851 \pm 65\end{array}$ & $\begin{aligned} & \text { LA } \\
& 1623 \pm 430 \\
& 1469 \pm 367 \\
& 788 \pm 197 \\
& 1070 \pm 267\end{aligned}$ & $\begin{array}{c}\text { RGP } \\
1515 \pm 260 \\
1322 \pm 228 \\
628 \pm 108 \\
809 \pm 140\end{array}$ & $\begin{array}{c}\text { RGA } \\
1213 \pm 102 \\
1034 \pm 97 \\
685 \pm 64 \\
845 \pm 88\end{array}$ \\
\hline $\begin{array}{l}\text { dans le rumen } \\
\text { a } 0 \mathrm{~h}(\mathrm{~g})\end{array}$ & $\begin{array}{l}\text { MF } \\
\text { MS } \\
P\end{array}$ & $\begin{array}{c}5191 \pm 875 \\
543 \pm 37 \\
474 \pm 1\end{array}$ & $\begin{array}{r}7290 \pm 925 \\
1059 \pm 176 \\
872 \pm 159\end{array}$ & $\begin{array}{c}8459 \pm 2264 \\
452 \pm 134 \\
322 \pm 119\end{array}$ & $\begin{array}{c}7941 \pm 170 \\
745 \pm 38 \\
632 \pm 44\end{array}$ \\
\hline $\begin{array}{l}\text { dans le rumen } \\
\text { à } 4 \mathrm{~h}(\mathrm{~g})\end{array}$ & $\begin{array}{l}\text { MF } \\
\text { MS } \\
P\end{array}$ & $\begin{array}{c}11393 \pm 498 \\
1501 \pm 117 \\
1171 \pm 60\end{array}$ & $\begin{array}{r}11937 \pm 862 \\
1776 \pm 253 \\
1426 \pm 174\end{array}$ & $\begin{array}{c}11793 \pm 2187 \\
1224 \pm 310 \\
885 \pm 238\end{array}$ & $\begin{array}{c}12132 \pm 1120 \\
1628 \pm 148 \\
1409 \pm 166\end{array}$ \\
\hline $\begin{array}{l}\text { disparue }(\mathrm{g} / \mathrm{h}) \\
\text { de } 0 \text { à } 4 \mathrm{~h}\end{array}$ & $\begin{array}{l}\text { MS } \\
P\end{array}$ & $\begin{array}{l}78 \\
39\end{array}$ & $\begin{array}{l}188 \\
129\end{array}$ & $\begin{array}{r}138 \\
62\end{array}$ & $\begin{array}{l}38 \\
17\end{array}$ \\
\hline $\begin{array}{l}\text { disparue }(\mathrm{g} / \mathrm{h}) \\
\text { de } 4 \text { a } 24 \mathrm{~h}\end{array}$ & $\begin{array}{l}\text { MS } \\
P\end{array}$ & $\begin{array}{l}48 \\
35\end{array}$ & $\begin{array}{l}36 \\
28\end{array}$ & $\begin{array}{l}39 \\
28\end{array}$ & $\begin{array}{l}44 \\
40\end{array}$ \\
\hline $\begin{array}{l}\text { disparue }(\mathrm{g} / \mathrm{h}) \\
\text { de } 0 \text { à } 24 \mathrm{~h}\end{array}$ & $\begin{array}{l}\text { MS } \\
P\end{array}$ & $\begin{array}{l}53 \\
36\end{array}$ & $\begin{array}{l}61 \\
45\end{array}$ & $\begin{array}{l}55 \\
34\end{array}$ & $\begin{array}{l}43 \\
35\end{array}$ \\
\hline
\end{tabular}

La vitesse de disparition de la MS et des particules du rumen, pendant les $4 \mathrm{~h}$ qui suivent la distribution du repas, est plus élevée pour la luzerne que pour le ray-grass parce que les légumineuses sont réduites plus facilement en fines particules et digérées plus rapidement dans le rumen. Elles peuvent donc être ingérées en plus grande quantité.

Je remercie le Dr Moseley (Grande-Bretagne) pour ses conseils concernant le dépouillement des résultats.

Grenet E., 1986. Reprod. Nutr. Dévelop., 26, 273-274. 